\title{
LigTMap: Ligand and structure-based target identification and activity prediction for small molecules
}

Faraz Shaikh ${ }^{1}$, Hio Kuan Tai ${ }^{1}$, Nirali Desai ${ }^{1,2}$, Shirley W. I. Siu ${ }^{1 *}$

${ }^{1}$ Department of Computer and Information Science, Faculty of Science and Technology, University of Macau, Avenida da Universidade, Taipa, Macau, China

${ }^{2}$ Division of Biological and Life Sciences, Ahmedabad University, India

*Corresponding Author:

E-mail: shirleysiu@um.edu.mo

Phone: +853 8822-4452

Fax: $+8538822-2426$ or +8533974285

\section{KEYWORDS}

Target prediction; binding affinity prediction; fingerprint similarity; binding interaction fingerprint; inverse docking; drug repurposing; PSOVina; random forest

\section{SUPPLEMENTARY INFORMATION}

The supplementary PDF contains additional material and method description, benchmark data and prediction results. 


\begin{abstract}
Motivation: Target prediction is a crucial step in modern drug discovery. However, existing experimental approaches to target prediction are time-consuming and costly.
\end{abstract}

Results: The LigTMap server provides a fully automated workflow to identify targets from 17 target classes with $>6000$ proteins. It is a hybrid approach, combining ligand similarity search with docking and binding similarity analysis, to predict putative targets. In the validation experiment, LigTMap achieved a top-10 success rate of almost 70\%, with an average precision rate of 0.34 . The class-specific prediction method improved the success rate further with enhanced precision. In an independent benchmarking test, LigTMap showed good performance compared to the currently best target prediction servers. LigTMap provides straightaway the PDB of a predicted target and the optimal ligand binding mode, which could facilitate structure-based drug design and the repurposing of existing drugs.

Availability: LigTMap is free for non-commercial use at https://cbbio.cis.um.edu.mo/LigTMap/. (145 words) 


\section{INTRODUCTION}

In recent years, the number of small natural and synthetic molecules, both real and virtual, has significantly increased (Hoffmann and Gastreich, 2019). One way to evaluate their potential for therapeutic application is to identify their disease-related molecular targets. Similarly, finding novel targets for existing drugs, i.e., drug repurposing, exposes new clinical applications of known drugs in less time and at lower cost in comparison to traditional approaches (Vanhaelen et al., 2017). On the other hand, novel drug targets may exhibit undesirable side effects and toxicity, which must be considered to improve the safety profiles of such agents (Wu et al., 2018). Despite technological advances, experimental methods to target identification remain laborious, expensive, and sometimes unsuccessful. Moreover, initial hypotheses on the potential target are typically required as the basis for the design of effective biochemical and genetic interaction experiments (Schenone et al., 2017).

Over the years, various in silico approaches have been developed to provide solutions to the target prediction problem (Agamah et al., 2019). Table S1 presents a list of some of these computational target prediction methods, highlighting their methodological strategies, employed datasets, and availability of online servers. These approaches can be broadly classified into three groups: ligandbased, structure-based, and hybrid (Mathai et al., 2019)(Sydow et al., 2019). The central notion of ligand-based approaches is that chemically similar compounds exhibit analogous biological activities (Matter, 1997). Thus, ligand-based methods extract chemical features of molecules using fingerprint algorithms to compare the similarities between the query compounds and the ligands with known activities (Matter, 1997). Despite their simplicity, with prior knowledge of ligands and their targets, ligand-based methods are effective and fast. Nevertheless, an undeniable 
disadvantage of ligand-based methods is that prediction for compounds with new chemical scaffolds is limited. Examples of ligand-based methods include SEA (Keiser et al., 2007), SuperPred (Nickel et al., 2014), PASS (Lagunin et al., 2000), and TarPred (Liu et al., 2014). To improve predictive performance, new methods have recently emerged which utilize supervised (such as HitPick (Liu et al., 2013) and Target Hunter (Wang et al., 2013)) and unsupervised machine learning (ML) (such as SPiDER (Reker et al., 2014)) to improve the model precision rate. Moreover, some earlier methods, including ChemProt (Kringelum et al., 2016) and SwissTargetPrediction (Daina et al., 2019), have also updated their search engines to ML models, which show greater effectiveness.

On the other hand, structure-based approaches utilize the available three-dimensional (3D) structural information of the target. They either apply docking to estimate the structural and chemical fitness of the query compound to the target or extract a set of pharmacophores from protein-ligand complexes and check whether the query compound matches well with the pharmacophores. In both cases, sufficient exploration of the ligand or protein conformational space is necessary. Consequently, structure-based approaches are costly and more time-consuming than the ligand-based methods. Several approaches of this type have been developed including TarFisDock (Li et al., 2006), PharmMapper (Wang et al., 2017), DRAR-CPI (Luo et al., 2011), PatchSearch (Rey et al., 2019), ACID (Wang et al., 2019), and Zhang (Zhang et al., 2019). However, few of them provide an online server, and in the ones that do, the numbers of searchable targets are limited. Finally, methods that combine both ligand and structural information, such as ChemMapper (Gong et al., 2013), can be utilized to predict more complex systems (Sydow et al., 2019). In addition to the typical protein or ligand data, other biological information, including 
protein sequence, protein-protein and protein-ligand interactions, and disease pathway, can be utilized to more reliably deduce the related target (Madhukar et al., 2019).

In the present study, a new, hybrid, fully automated target prediction workflow called LigTMap was developed to predict targets for query compounds. Here, ligand similarity search was proposed as the first step to short-list the putative targets. We investigated the influence of different fingerprints and cutoff values for effective target selection. In the second step, the binding mode of the query compound at a putative target was predicted by molecular docking and its binding mode was compared to the binding mode of the crystal ligand. Ranking of the targets was based on the combined score from both the ligand and binding similarity scores. To assess the performance of LigTMap, we compared it to four existing servers using a set of manually curated benchmark compounds.

\section{MATERIALS \& METHODS}

\section{Dataset}

The ligand and protein structures, as well as their experimental activity data in $\mathrm{Ki}, \mathrm{Kd}$, or IC50, were obtained from the PDBbind database (version 2017) (Wang et al., 2004). This annually updated database has been widely used as the benchmark for the comparison of protein-ligand docking programs and for the assessment of scoring functions. For the purpose of target prediction, we labeled each PDB from the dataset with its actual target class by referring to the PDB database (Berman et al., 2000) and the available literature to ensure correctness. In total, 17 target classes were processed; among them, 11 were human protein targets and 6 were disease targets. The former included the kinase, transferase, beta-secretase, hydrolase, ligase, and isomerase enzymes 
as well as an anticoagulant, a bromodomain (BRD), peroxisome, estrogen, and carbonic anhydrase (CA), while the latter included the human immunodeficiency virus (HIV), tuberculosis (TB), hepatitis $\mathrm{C}$ virus (HCV), influenza, Helicobacter pylori (H. pylori), and diabetes. Except for diabetes, the disease targets were nonhuman protein targets that originate from viruses or bacteria. Descriptions about the preparation of the data for fingerprint generation and docking are provided in the Supplementary Materials and Method section. In summary, there are totally 6313 proteins in the 17 datasets as presented in Table S2.

For model validation, approximately $20 \%$ of the data from each target class was designated as the validation data, and the remaining was the training data. In total, there were 1251 ligands in the validation set. To test the performance of LigTMap independently, newly identified compounds with experimentally validated targets were searched in the available medicinal chemistry journals. To ensure that these compounds were not already included in our datasets or in the datasets of the methods used for comparison only reports published in 2018 and later were considered. In total, 98 compounds were obtained for 7 target classes, including kinase, ligase, BRD, CA, betasecretase, HIV, and TB. It should be noted that the benchmark data also contained compounds for multiple targets - kinase and BRD (categorized into the kinase class) as well as TB and kinase (categorized into the TB class). Moreover, for 10 target classes, new compounds were not found in the literature, and thus, they were not included in the benchmark experiments. 


\section{Target Prediction Workflow}

The workflow of the LigTMap target prediction is illustrated in Figure 1. It consists of five steps:

1. For a query compound, a set of potential targets is selected based on fingerprint similarities to the crystal ligands. Multiple fingerprints (ㅆorgan, $\underline{\text { MACCS, }}$ Daylight) are generated, and the ligand similarity score $\left(T_{L}\right)$ is taken as an average of either three MMD or two MM fingerprint Tanimoto coefficients, depending on whether the run is for all-target class prediction or class-specific prediction. The Tanimoto coefficient can be computed as follows (Delaney, 1996):

$$
T=\frac{N_{a b}}{N_{a}+N_{b}-N_{a b}}
$$

where $N_{a}$ and $N_{b}$ are the numbers of 1-bit in the fingerprints of ligand $a$ and ligand $b$, respectively. $N_{a b}$ denotes the number of 1-bit common to both ligands. A cutoff value for $T_{L}$ is used as the target selection criteria; the default is 0.4 .

2. For each potential target, molecular docking is performed using PSOVina2 (Tai et al., 2018) to predict the most optimal binding mode of the compound in the ligand-binding pocket.

3. A binding interaction fingerprint (IFP) of the compound is generated based on the predicted binding mode. The established IFP is compared with the IFP of the crystal ligand using the Tanimoto coefficient to obtain the binding similarity score $\left(T_{B}\right)$ (Eq. 1).

4. For each potential target class, the compound binding activity is predicted using the classspecific random forest $(\mathrm{RF})$ model based on the Avalon fingerprint.

5. Finally, all prediction results are consolidated, and the protein targets are ranked based on the combined score LigTMap score $=0.7 T_{L}+0.3 T_{B}$. 


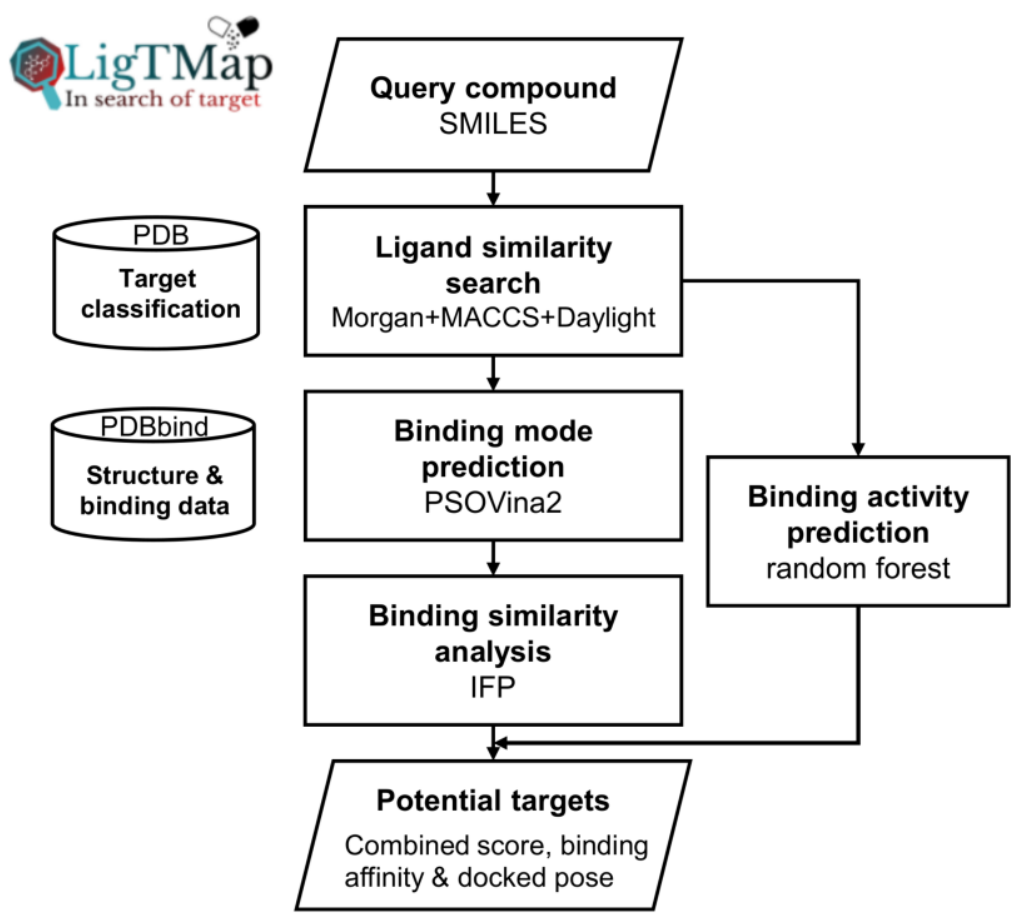

Figure 1. Workflow of LigTMap for all-target class prediction. For class-specific prediction, MM is used instead of MMD in the ligand similarity search.

\section{Target Prediction Performance Measures}

A predicted target is considered correct if its name matches the name of the experimental target of a compound. Moreover, in the case of a multi-target compound, a predicted target matching with any of the known targets is considered correct. When testing a set of ligands, we computed the success rate as the fraction of ligands in the set predicted correctly within top $N \in\{1,5,10\}$ of the output list. In addition, for each ligand prediction, we computed the recall, precision, and $F 1$ scores taking the top $N$ targets as predicted positives and all correct targets as actual positives:

$$
\text { recall }=\frac{T P}{T P+F N}
$$




$$
\begin{gathered}
\text { precision }=\frac{T P}{T P+F P} \\
F 1=2 \cdot \frac{\text { precision } \cdot \text { recall }}{\text { precision }+ \text { recall }}
\end{gathered}
$$

Thus, recall measures the proportion of correctly predicted targets among all correct targets, while precision indicates the proportion of correctly predicted targets within top $N$. $F 1$ provides a single estimate combining recall and precision.

\section{Ligand Binding Activity Prediction}

Once a target is identified, it is desirable to obtain an estimation of the compound binding activity toward the target. In the current method, the binding activity (pKi/Kd/IC50) is predicted employing a class-specific ML model. Four ML algorithms based on the Avalon fingerprint were tested, including RF, support vector machines, gradient boosted tree, and $K$-nearest neighbor algorithms. RF was selected, as the preliminary tests using five selected target class datasets indicated that, in comparison to the other algorithms, it produced superior results. To prevent the issue of information leak (Wainer and Cawley, 2018), nested cross-validation (CV) (Stone, 1976) was used to train and optimize the RF models. The detail of the nested CV for model training-andoptimization is presented in the Supplementary Materials and Methods section. 


\section{RESULTS AND DISCUSSION}

LigTMap is a ligand- and structure-based method for target prediction. It consists of five key steps: 1) ligand similarity search to identify potential targets and the associated target class, 2) prediction of ligand binding modes toward the potential targets, 3) assessment of binding similarity to the crystal ligand binding modes, 4) prediction of class-specific binding activity, and 5) consolidation of results and ranking.

\section{Selection of the Fingerprint Algorithm for Ligand Similarity Search}

Previous studies have shown that the choice of the fingerprint algorithm is crucial for the success of ligand-based target prediction. Furthermore, combining multiple fingerprints further improves the success rates of target prediction models (Wang et al., 2016) (Kogej et al., 2006). To establish an optimal combination of fingerprints for the ligand similarity search, we tested six different fingerprint algorithms, i.e., Morgan, MACCS, Daylight, AtomPairs, Torsion, and Pharmacophore, as well as their combinations for target prediction using the validation set. Supplementary Figure S2 shows the distribution of the number of ligands with correctly predicted targets, which ranked within the top 1,5 , and 10 in the output of the ligand similarity search. The figure also indicates the number of ligands for which targets ranked below 10 but were still in the prediction list. Based on the obtained results, it is clear that when the cutoff decreased from 1.0 to 0.1 , the number of correctly predicted targets increased until reaching a certain cutoff value, at which the top 1/5/10 results remained relatively stable. This "optimal" range of cutoff values varied for different fingerprints. For Morgan, AtomPairs, and Torsion, it was determined at $0.1-0.3$; for MACCS and Daylight, it was 0.1-0.5; and for Pharmacophore, it was 0.1-0.2. When extracting the potential targets in LigTMap, if the cutoff is set too high, actual targets may be discarded too early. 
Conversely, if the cutoff is set too low, too many false positives are included, causing excessive computations in the subsequent steps. Among the six fingerprints, Morgan, MACCS, and Daylight were considered as the best options. Importantly, MACCS and Daylight included correct targets with high cutoff, while Morgan predicted correct targets in top 10 for most ligands. On the other hand, AtomPairs, Torsion, and Pharmacophore exhibited worse performance or required low cutoff.

Subsequently, we also tested combinations of fingerprints, i.e., Morgan+MACCS (MM) and Morgan+MACCS+Daylight (MMD) for target prediction, where the average of the component scores was taken as the ligand similarity score. As shown in Supplementary Figure S3, the combined fingerprints performed better than MACCS and Daylight alone with improved correct top 10 predictions. In addition, they achieved similar performances as Morgan, however, with increased optimal cutoff range (between 0.1 and 0.4). Consequently, we considered both combined fingerprints in further experiments and took the borderline cutoff of 0.4 as default in the LigTMap workflow.

\section{LigTMap Target Prediction Performance Evaluation}

Predictive performance of the entire LigTMap workflow was assessed utilizing both the validation and benchmark sets. Because the binding IFP calculation was computationally expensive, we tested target classes from the validation set of which benchmark data was also available. These included kinase, beta-secretase, BRD, CA, ligase, HIV, and TB. Totally, 733 ligands were tested from the validation set and 98 from the benchmark set. The entire target prediction workflow was 
run comprising the MM or MMD for the ligand similarity search with IFP based binding similarity analysis. Target ranking was based on the LigTMap score that was obtained as a weighted sum of the ligand similarity and binding similarity scores. Furthermore, according to the SEA target prediction study, target class-specific models improve the prediction precision rate in ligand-based methods (Wang et al., 2016). To verify this hypothesis, we compared the results from the all-target class prediction to those from the class-specific prediction. In the CS experiment, each ligand was predicted for its target class only, and thus, the output contained only targets from this class. Table 1 shows the overall performance of LigTMap in the conducted experiments.

For all-target class prediction, the LigTMap score with MMD achieved higher top-5 and top-10 success rates than that with MM in both validation and benchmark experiments. It achieved an average top-10 success rate of almost $70 \%$, with an average precision rate of 0.34 and recall of 0.26. Notably, the comparison between all-target class experiments (LigTMap score with MM or MMD) and CS experiments (with MM-CS or MMD-CS) revealed significant improvement in all measures. This is presumably due to the exclusion of "off-targets" from the prediction list. Moreover, the comparison between MM-CS and MMD-CS shows that LigTMap score with MMCS has a 1\%-5\% higher success rate and 15\%-35\% improvement in recall with comparable precision. Meanwhile, comparing to MMD, LigTMap score with MM-CS improved the top-1 success rate by $27 \%$ and the top- 10 success rate by $17 \%$, with $>50 \%$ increase in precision. Overall, the LigTMap score with MMD outperformed that with MM in all-target class predictions; however, LigTMap score with MM-CS surpassed MMD-CS in the CS predictions. 
Table 1. Overall performance of LigTMap using MM or MMD as ligand similarity search, for all-target class prediction or class-specific (CS) prediction.

\begin{tabular}{|l|c|c|c|c|c|c|}
\hline & \multicolumn{3}{|c|}{ Average Success Rate } & \multicolumn{3}{c|}{ Average (Top 10) } \\
\hline & Top 1 & Top 5 & Top 10 & Precision & Recall & F1 Score \\
\hline Validation Set & 0.56 & 0.68 & 0.72 & 0.35 & 0.27 & 0.23 \\
\hline MM & 0.53 & 0.70 & 0.73 & 0.35 & 0.25 & 0.20 \\
\hline MMD & 0.63 & 0.75 & 0.77 & 0.55 & 0.52 & 0.45 \\
\hline MM-CS & 0.61 & 0.74 & 0.76 & 0.54 & 0.38 & 0.35 \\
\hline MMD-CS & & & & & & 0.27 \\
\hline Benchmark Set & 0.40 & 0.59 & 0.63 & 0.29 & 0.26 \\
\hline MM & 0.44 & 0.64 & 0.65 & 0.33 & 0.27 & 0.24 \\
\hline MMD & 0.60 & 0.74 & 0.84 & 0.53 & 0.52 & 0.65 \\
\hline MM-CS & 0.57 & 0.73 & 0.82 & 0.55 & 0.45 & 0.44 \\
\hline MMD-CS & & & & & & \\
\hline
\end{tabular}

Table 2 demonstrates the detailed predictive performance of LigTMap for each target class. Among the seven target classes, in the validation set, the highest success rate was achieved for beta-secretase (>0.9), followed by BRD, HIV ( 0.8), CA and ligase ( 0.7), kinase, and TB ( 0.5). For the benchmark set, LigTMap performed exceptionally for CA, beta-secretase, and BRD (>0.9). It also showed good performance for HIV (>0.8) and moderate for kinase ( 0.7). LigTMap failed to find correct targets for ligase and TB ligands. In consistent with Table 1, LigTMap score with MMD performed better than MM in all-target class experiments for most targets in terms of the top 10 success rate, in expense of the reduced F1 score. As a target prediction method, successful identification of the query compounds is of great importance; thus, LigTMap score with MMD is suitable. For compounds the target class of which is known but not the proteins, MM-CS is more optimal for finding the correct target within the class. The prediction results of all benchmark 
compounds with their experimental targets, predicted targets, PDB IDs, and ranks of the first true targets are provided in Tables S3-S9 of the Supplementary Information.

Table 2. Predictive performance for each target class in all-target class and class-specific experiments.

\begin{tabular}{|c|c|c|c|c|c|c|c|c|}
\hline & \multicolumn{2}{|c|}{$\begin{array}{l}\text { LigTMap score } \\
\text { (MM) }\end{array}$} & \multicolumn{2}{|c|}{$\begin{array}{l}\text { LigTMap score } \\
\text { (MMD) }\end{array}$} & \multicolumn{2}{|c|}{$\begin{array}{c}\text { LigTMap score } \\
\text { (MM-CS) }\end{array}$} & \multicolumn{2}{|c|}{$\begin{array}{c}\text { LigTMap score } \\
\text { (MMD-CS) }\end{array}$} \\
\hline & Top 10 & F1 Score & Top 10 & F1 Score & Top 10 & F1 Score & Top 10 & F1 Score \\
\hline \multicolumn{9}{|l|}{ Validation Set } \\
\hline Kinase & 0.52 & 0.20 & 0.54 & 0.16 & 0.55 & 0.32 & 0.56 & 0.19 \\
\hline Beta-secretase & 0.97 & 0.29 & 0.97 & 0.23 & 1.00 & 0.54 & 1.00 & 0.42 \\
\hline Bromodomain & 0.79 & 0.25 & 0.82 & 0.25 & 0.91 & 0.63 & 0.88 & 0.55 \\
\hline $\begin{array}{l}\text { Carbonic } \\
\text { anhydrase }\end{array}$ & 0.74 & 0.19 & 0.72 & 0.23 & 0.74 & 0.29 & 0.72 & 0.28 \\
\hline Ligase & 0.72 & 0.34 & 0.72 & 0.23 & 0.72 & 0.72 & 0.72 & 0.4 \\
\hline HIV & 0.79 & 0.14 & 0.83 & 0.13 & 0.83 & 0.18 & 0.85 & 0.17 \\
\hline TB & 0.49 & 0.53 & 0.21 & 0.20 & 0.62 & 0.58 & 0.44 & 0.42 \\
\hline \multicolumn{9}{|l|}{ Benchmark Set } \\
\hline Kinase & 0.67 & 0.15 & 0.72 & 0.13 & 0.89 & 0.23 & 0.72 & 0.22 \\
\hline Beta-secretase & 0.89 & 0.42 & 1.00 & 0.28 & 0.95 & 0.49 & 0.95 & 0.30 \\
\hline Bromodomain & 0.89 & 0.27 & 0.95 & 0.33 & 0.95 & 0.72 & 0.95 & 0.70 \\
\hline $\begin{array}{l}\text { Carbonic } \\
\text { anhydrase }\end{array}$ & 1.00 & 0.54 & 1.00 & 0.60 & 1.00 & 0.81 & 1.00 & 0.85 \\
\hline Ligase & 0.00 & 0.00 & 0.00 & 0.00 & 0.20 & 0.00 & 0.20 & 0.00 \\
\hline HIV & 0.80 & 0.29 & 0.80 & 0.31 & 1.00 & 0.83 & 1.00 & 0.93 \\
\hline TB & 0.18 & 0.13 & 0.09 & 0.01 & 0.91 & 1.49 & 0.91 & 0.08 \\
\hline
\end{tabular}




\section{Comparison to the State-of-the-Art Target Prediction Methods}

The benchmark dataset was tested using four state-of-the-art target prediction servers, i.e., SwissTargetPrediction (Daina et al., 2019), SEA (Wang et al., 2016), SuperPred (Nickel et al., 2014), and HitPick (Liu et al., 2013). As these servers mainly provide prediction for human targets, we excluded nonhuman targets from the comparative study. In total, 77 ligands for kinase, betasecretase, CA, ligase, and BRD were evaluated. As benchmark data run with all classes, LigTMap score with MMD was used and results are shown in Table 3. LigTMap exhibited the highest top10 success rates of $86 \%$, followed by SEA (83\%), and SwissTargetPrediction (78\%). Moreover, it outperforms SuperPred and HitPick in all top-1, top-5, and top-10 success rates. SwissTargetPrediction has the highest top-1 success rate of $66 \%$.

Table 3. The number of top-1, top-10, and failure predictions in the benchmark set for five target prediction servers.

\begin{tabular}{|c|c|c|c|c|c|c|c|c|c|c|c|c|c|c|c|}
\hline & \multicolumn{3}{|c|}{ SwissTargetPrediction } & \multicolumn{3}{|c|}{ SEA } & \multicolumn{3}{|c|}{ SuperPred } & \multicolumn{3}{|c|}{ HitPick } & \multicolumn{3}{|c|}{ LigTMap } \\
\hline & $\begin{array}{c}\text { Top } \\
1\end{array}$ & $\begin{array}{c}\text { Top } \\
10\end{array}$ & Fail & $\begin{array}{c}\text { Top } \\
1\end{array}$ & $\begin{array}{c}\text { Top } \\
10\end{array}$ & Fail & $\begin{array}{c}\text { Top } \\
1\end{array}$ & $\begin{array}{c}\text { Top } \\
10\end{array}$ & Fail & $\begin{array}{c}\text { Top } \\
1\end{array}$ & $\begin{array}{c}\text { Top } \\
10\end{array}$ & Fail & $\begin{array}{c}\text { Top } \\
1\end{array}$ & $\begin{array}{c}\text { Top } \\
10\end{array}$ & Fail \\
\hline Beta-secretase & 16 & 19 & 0 & 17 & 19 & 0 & 9 & 19 & 0 & 6 & 6 & 13 & 15 & 19 & 0 \\
\hline Bromodomain & 17 & 19 & 0 & 19 & 19 & 0 & 0 & 0 & 19 & 0 & 0 & 19 & 14 & 18 & 1 \\
\hline Kinase & 10 & 13 & 5 & 1 & 18 & 0 & 0 & 0 & 18 & 10 & 10 & 8 & 3 & 13 & 5 \\
\hline $\begin{array}{l}\text { Carbonic } \\
\text { anhydrase }\end{array}$ & 8 & 9 & 7 & 4 & 8 & 8 & 6 & 7 & 8 & 1 & 5 & 8 & 13 & 16 & 0 \\
\hline Ligase & 0 & 0 & 5 & 0 & 0 & 5 & 0 & 0 & 5 & 0 & 0 & 5 & 0 & 0 & 5 \\
\hline Total & 51 & 60 & 17 & 41 & 64 & 13 & 15 & 27 & 50 & 17 & 24 & 53 & 45 & 66 & 11 \\
\hline$(\%)$ & 66 & 78 & 22 & 53 & 83 & 17 & 19 & 35 & 65 & 22 & 31 & 69 & 59 & 86 & 14 \\
\hline
\end{tabular}


Regarding the predictions for each target class, all beta-secretase ligands were successfully predicted by SwissTargetPrediction, SEA, and LigTMap. The same outcomes were noted for BRD, apart from LigTMap, which had one failure. For kinase, SEA successfully predicted all ligands; however, SwissTargetPrediction and LigTMap resulted in five failures. Furthermore, for CA, LigTMap was the only method, which predicted all ligands, with the remaining four methods predicting just half of the cases. Ligase proved to be the most challenging target in the benchmarking experiment; no methods provided successful prediction for this target class. Based on the conducted analyses, it can be concluded that LigTMap reached the state-of-the-art performance and, in some target classes, outperformed the existing methods.

\section{Performance of Ligand Binding Activity Prediction}

The predictive performance of all-target class-specific RF models using the core set is presented in Table 4. Two metrics were used to measure the performance of the models, i.e., the Pearson's correlation coefficient $(\mathrm{R})$ and the RMSE between the experimentally measured binding constants and the predicted values. Both coefficients were obtained by averaging from the test folds in the outer $\mathrm{CV}$ loop. The nested $\mathrm{CV}$ run was performed 10 times for each target, and the average performance and standard deviation were reported. Based on the obtained outcomes, we observed that $\mathrm{R}$ ranges between 0.5 to 0.8 for different target classes, with the HIV model achieves the highest correlation of 0.81 and the estrogen model the lowest correlation of 0.47 . Overall, the average performance gives a correlation of 0.61 and RMSE of $1.23(-\log \mathrm{M})$. 
Table 4. Cross-validation performance of 17 target class-specific RF activity prediction models on the core dataset.

\begin{tabular}{|l|c|c|}
\hline Target Class & $\begin{array}{c}\text { Pearson's Correlation } \\
\text { Coefficient }\end{array}$ & $\begin{array}{c}\text { RMSE } \\
(-\log \mathbf{~ M})\end{array}$ \\
\hline HIV & $0.81 \pm 0.01$ & $1.28 \pm 0.03$ \\
\hline Beta-secretase & $0.75 \pm 0.01$ & $0.97 \pm 0.03$ \\
\hline Ligase & $0.73 \pm 0.01$ & $1.26 \pm 0.03$ \\
\hline TB & $0.67 \pm 0.02$ & $1.24 \pm 0.03$ \\
\hline Transferase & $0.67 \pm 0.01$ & $1.30 \pm 0.02$ \\
\hline Kinase & $0.66 \pm 0.06$ & $1.18 \pm 0.01$ \\
\hline HCV & $0.65 \pm 0.02$ & $1.22 \pm 0.04$ \\
\hline Bromodomain & $0.65 \pm 0.02$ & $0.93 \pm 0.02$ \\
\hline Carbonic anhydrase & $0.64 \pm 0.02$ & $1.23 \pm 0.04$ \\
\hline Anticoagulant & $0.63 \pm 0.02$ & $1.37 \pm 0.03$ \\
\hline Hydrolase & $0.62 \pm 0.02$ & $1.42 \pm 0.03$ \\
\hline Helicobacter pylori & $0.60 \pm 0.11$ & $1.44 \pm 0.19$ \\
\hline Influenza & $0.59 \pm 0.05$ & $1.77 \pm 0.08$ \\
\hline Diabetes & $0.53 \pm 0.03$ & $1.05 \pm 0.03$ \\
\hline Isomerase & $0.53 \pm 0.03$ & $1.38 \pm 0.01$ \\
\hline Peroxisome & $0.48 \pm 0.07$ & $0.99 \pm 0.10$ \\
\hline Estrogen & $0.47 \pm 0.07$ & $1.12 \pm 0.05$ \\
\hline & $\mathbf{0 . 6 1}$ & $\mathbf{1 . 2 3}$ \\
\hline
\end{tabular}

The class-specific RF models were further assessed using the benchmark set. As shown in Table 5 , the average correlation is 0.63 , while RMSE is 1.26 , which is in good agreement with the CV result. The two cases that are found different between the benchmark and validation results are the beta-secretase (benchmark/validation $0.31 / 0.75)$ and kinase $(0.18 / 0.66)$. For the beta-secretase, the experimental values of the test compound concentrated in a narrow range of 7.6-8.2, whereas the 
prediction gave a range of 7.2-9. Despite the poor correlation, the RMSE for beta-secretase is low (only 0.49 ), suggesting that the predicted values were reasonably close to the experimental ones.

Table 5. Test performance of 7 target class-specific RF activity prediction models on the benchmark dataset.

\begin{tabular}{|l|c|c|}
\hline Target Class & $\begin{array}{c}\text { Pearson's Correlation } \\
\text { Coefficient }\end{array}$ & $\begin{array}{c}\text { RMSE } \\
\text { (-log M) }\end{array}$ \\
\hline Bromodomain & 0.95 & 0.65 \\
\hline Ligase & 0.88 & 0.99 \\
\hline HIV & 0.83 & 0.44 \\
\hline Carbonic anhydrase & 0.57 & 1.92 \\
\hline TB & 0.72 & 2.71 \\
\hline Beta-secretase Average & 0.31 & 0.49 \\
\hline Kinase & 0.18 & 1.62 \\
\hline & $\mathbf{0 . 6 3}$ & $\mathbf{1 . 2 6}$ \\
\hline
\end{tabular}

In the kinase class, some of the compounds were identified experimentally to target two different kinase proteins (PLK1 and ALK), and some also targeted another class, i.e., bromodomain BRD4. Taking the average of the PLK1 and ALK activity values and comparing them to the predicted values gave a poor correlation of 0.18 and RMSD of 1.62. Nevertheless, the predictions correlated better with PLK1 alone, giving an improved correlation of 0.61 and RMSD of 0.85 (see Table S14). However, worse outcomes were noted when the predictions were correlated with ALK alone (correlation of -0.33 and RMSD of 1.11). As none of the dual-class compounds were identified for 
$\mathrm{BRD}$, the predicted activity using the BRD model poorly correlated with the experimental data for BRD4 (correlation of -0.09 and RMSD of 1.17).

In the case of $\mathrm{TB}$, although all compounds were identified to target both the bacterial $\mathrm{TB}$ (MtbAdok) and human kinase (hAdoK), only the TB model correlated reasonably with the MtbAdok activity (correlation of 0.72 and RMSD of 2.71). The kinase model did not correlate with hAdoK (correlation of 0.24 and RMSD of 5.42).

The activity prediction results of the benchmark set are listed in Tables S9-S15.

\section{Case Study of the HIV Drug Target Prediction}

Structure-based drug design has played an important role in the anti-HIV drug search. Since the first discovery of HIV 36 years ago (Gallo et al., 1983) more than 30 drugs for the treatment of AIDS have been developed and identified to target all 7 life cycles of the virus (FDA-Approved HIV Medicines). The fast development of novel anti-HIV agents targeting multiple targets can be attributed to the availability of the 3D structures of the HIV proteins. In the PDBbind dataset alone, there are currently 580 crystal complexes of the HIV proteins as well as their ligands. The structural information, together with accurate experimental activity data of true binders, can be

exploited to construct highly accurate predictive HIV models to support the hits discovery and lead optimization. In the present study, the developed predictive RF model for HIV yields an average correlation of 0.81 with RMSE of $1.28(-\log \mathrm{M})$ in the nested CV assessment (see Table 4). Further evaluation of the RF model was performed using 10 novel compounds reported by Pribut et al. (Pribut et al., 2019). According to their study, aryl substituted benzimidazolones were 
experimentally validated to exhibit inhibitory effects against the HIV-1 non-nucleoside reverse transcriptase, with the reported pIC50 values in the range of 4.87-7.58. The binding modes of these compounds were studied by molecular docking using the receptor structures PDB 2jle and 2fr2. Notably, our RF model for HIV displayed remarkable performance in predicting the activities of these 10 compounds, yielding a correlation of 0.83 and RMSE of 0.44 (see Table 5).

Furthermore, for comparison, we also tested the new compounds using two recently released online servers for anti-HIV biological activity prediction, namely, AntiHIV-Pred (Stolbov et al., 2019) and HIVprotI (Qureshi et al., 2018). Their methods employed large-scale experimental data extracted from the ChEMBL database and their prediction models are ligand-based and HIV protein-specific. Users can select the prediction target as HIV protease, reverse transcriptase, integrase, REV (AntiHIV-Pred only), or TAT (AntiHIV-Pred only). Surprisingly, the AntiHIVPred server reported that the evaluated compounds were in the non-applicable domain, regardless of the selected target; therefore, no activity values were predicted. On the other hand, the HIVprotI server successfully returned prediction results for nine compounds against the reverse transcriptase target. However, poor correlation of 0.56 was obtained, which was significantly lower than the originally reported correlation of 0.76 (Qureshi et al., 2018). Figure S5 showed a comparison of the anti-HIV inhibitor predictions by HIVprotI and LigTMap, indicating that LigTMap performed superior in activity predictions. 

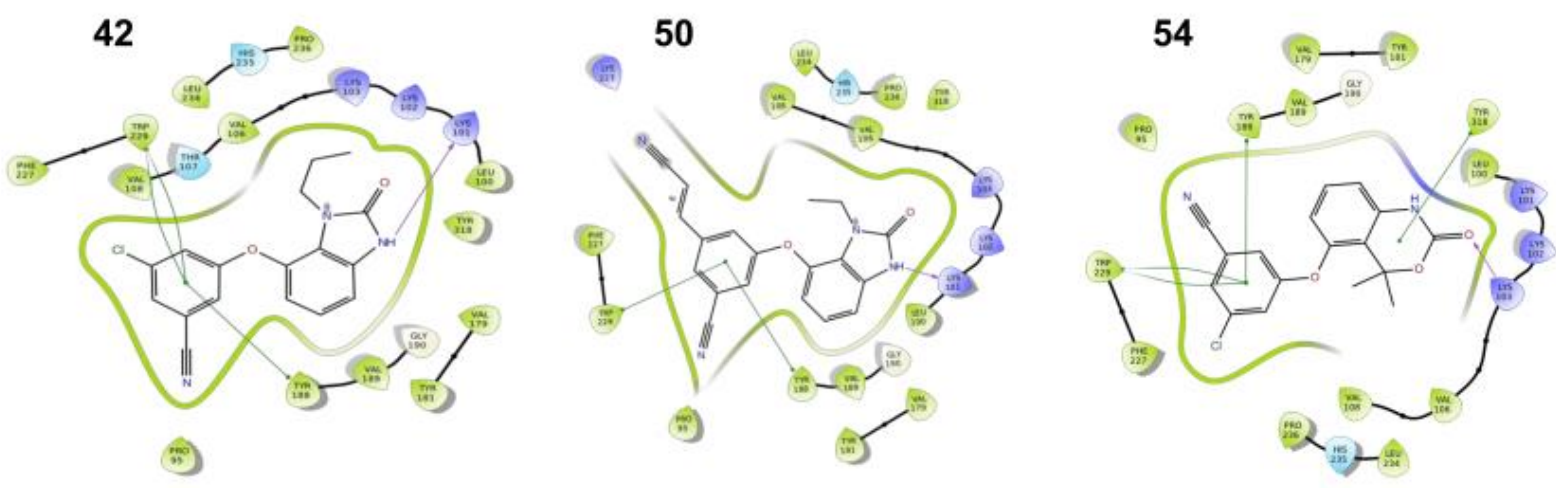

Figure 2. Predicted binding modes of three known anti-HIV ligands by PSOVina2 in LigTMap. Images were prepared using Schrödinger Maestro.

We compared the binding modes predicted by PSOVina2 in LigTMap to the previously reported binding modes (Pribut et al., 2019). The three compounds were evaluated: 42, 50, and 54. Previously, these compounds were lead optimized from the non-nucleoside reverse transcriptase inhibitor (PDB ID 2jle). Remarkably, LigTMap was able to predict the correct target for the three compounds in the top 1. As shown in Figure 2, our predicted binding modes matched closely to the reported protein-ligand interaction patterns. For instance, the binding of the second-generation benzimidazole inhibitor (compound $\mathbf{4 2}$ ) was reported to involve two pi-pi interactions with the Tyr188 and Trp229 residues, and a hydrogen bond to Lys101. The ligand docked by LigTMap retained an analogous binding mode, also predicting two pi-pi interactions and a hydrogen bond as the major contributors to the ligand binding. The reported binding modes of $\mathbf{5 0}$ involved the Lys101, Tyr188, Lys223, and Tyr229 amino acids, while Lys101, Tyr188, and Tyr229 participated in the interactions with compound $\mathbf{5 4}$. Notably, all of them were correctly predicted by LigTMap. 


\section{Features of the LigTMap Server}

The LigTMap server is free for non-commercial use at https://cbbio.cis.um.edu.mo/LigTMap/. The server accepts queries for multiple compound predictions (maximum of 20 for a batch submission) for both human and nonhuman target classes. The output of the target prediction displays the name of the predicted target, its PDB ID, the ligand similarity score, binding similarity score, predicted activity value, docking score, and docking pose determined using PSOVina2. In Table 6, the LigTMap server is compared to the existing state-of-the-art target prediction servers with respect to their target scope and prediction output.

Table 6. Comparison of various features of target prediction servers.

\begin{tabular}{|c|c|c|c|c|c|}
\hline Server Feature & SEA & $\begin{array}{l}\text { SwissTarget } \\
\text { Prediction }\end{array}$ & SuperPred & HitPick & LigTMap \\
\hline \multicolumn{6}{|l|}{ Target Scope } \\
\hline $\begin{array}{l}\text { Predict human } \\
\text { targets }\end{array}$ & Yes & Yes & Yes & Yes & Yes \\
\hline $\begin{array}{l}\text { Predict } \\
\text { nonhuman (or } \\
\text { disease) targets }\end{array}$ & No & Yes (few) & No & No & Yes \\
\hline \multicolumn{6}{|l|}{ Prediction } \\
\hline $\begin{array}{l}\text { Support input of } \\
\text { multiple compounds }\end{array}$ & No & No & No & Yes & Yes \\
\hline Target name & Yes & Yes & Yes & Yes & Yes \\
\hline Target PDB & No & No & Yes & No & Yes \\
\hline Biological activity & No & No & No & No & Yes \\
\hline Binding mode & No & No & No & No & Yes \\
\hline $\begin{array}{l}\text { External links to } \\
\text { target related } \\
\text { information }\end{array}$ & $\overline{Z I N C}$ & $\begin{array}{c}\text { Uniprot, } \\
\text { GeneCard }\end{array}$ & $\begin{array}{c}\text { Uniprot, } \\
\text { BindingDB, } \\
\text { RefSeq, etc. }\end{array}$ & GeneCard & PDB \\
\hline
\end{tabular}




\section{CONCLUSION}

Target prediction of small molecules is a crucial step in drug discovery and study of disease mechanisms. The existing computational approaches to target prediction are limited in terms of availability, functionality, and accuracy. In the current work, we present LigTMap, a new target prediction method developed to predict 17 target classes, including human and nonhuman protein targets. It is a multistage prediction workflow, which combines the ligand similarity search with docking and binding similarity analysis to accurately identify protein targets. Extensive experiments utilizing validation and benchmark sets revealed that LigTMap (MMD) achieved a top-10 success rate of almost $70 \%$, with an average precision rate of 0.34 . This performance is good as compared to the current best prediction servers SwissTargetPrediction and SEA. Classspecific target prediction of LigTMap (MM-CS) improved the top-1 success rate by $27 \%$ and the top-10 success rate by $17 \%$, with $>50 \%$ increase in precision. Hence, LigTMap is a new, reliable method for target prediction of novel ligands. Furthermore, it can identify with a higher success rate for ligands whose target class is known but the actual targets are still unknown.

The current version of LigTMap is limited to target classes prediction. For future work, other large compound databases, such as ChEMBL and ZINC, as well as protein-ligand interaction databases, e.g., STITCH, will be exploited to expand the target class coverage and enhance the prediction accuracy. 


\section{FUNDING}

This work was supported by the University of Macau (grant no. MYRG2017-00146-FST).

\section{ACKNOWLEGEMENTS}

The authors thank the support of the Faculty of Science and Technology and the Information and Communication Technology Office of University of Macau for providing the computing facilities.

\section{REFERENCES}

Agamah,F.E. et al. (2019) Computational/in silico methods in drug target and lead prediction. Brief. Bioinform., bbz103.

Berman,H.M. et al. (2000) The Protein Data Bank. Nucleic Acids Res., 28, 235-242.

Daina,A. et al. (2019) SwissTargetPrediction: updated data and new features for efficient prediction of protein targets of small molecules. Nucleic Acids Res., 47, W357-W364.

Delaney,J.S. (1996) Assessing the ability of chemical similarity measures to discriminate between active and inactive compounds. Mol. Divers., 1, 217-222.

FDA-Approved HIV Medicines https://aidsinfo.nih.gov/understanding-hiv-aids/fact-sheets/21/58/fdaapproved-hiv-medicines/.

Gallo,R.C. et al. (1983) Isolation of human T-cell leukemia virus in acquired immune deficiency syndrome (AIDS). Science (80), 220, 865-867.

Gong,J. et al. (2013) ChemMapper: a versatile web server for exploring pharmacology and chemical structure association based on molecular 3D similarity method. Bioinformatics, 29, 1827-1829. 
Hoffmann,T. and Gastreich,M. (2019) The next level in chemical space navigation: going far beyond enumerable compound libraries. Drug Discov. Today, 24, 1148-1156.

Keiser,M.J. et al. (2007) Relating protein pharmacology by ligand chemistry. Nat. Biotechnol., 25, 197206.

Kogej,T. et al. (2006) Multifingerprint based similarity searches for targeted class compound selection. $J$. Chem. Inf. Model., 46, 1201-1213.

Kringelum,J. et al. (2016) ChemProt-3.0: a global chemical biology diseases mapping. Database, bav123.

Lagunin,A. et al. (2000) PASS: prediction of activity spectra for biologically active substances. Bioinformatics, 16, 747-748.

Li,H. et al. (2006) TarFisDock: a web server for identifying drug targets with docking approach. Nucleic Acids Res., 34, W219-W224.

Liu,X. et al. (2013) HitPick: A web server for hit identification and target prediction of chemical screenings. Bioinformatics, 29, 1910-1912.

Liu,X. et al. (2014) In Silicotarget fishing: addressing a "Big Data" problem by ligand-based similarity rankings with data fusion. J. Cheminform., $6,33$.

Luo,H. et al. (2011) DRAR-CPI: a server for identifying drug repositioning potential and adverse drug reactions via the chemical-protein interactome. Nucleic Acids Res., 39, W492-W498.

Madhukar,N.S. et al. (2019) A Bayesian machine learning approach for drug target identification using diverse data types. Nat. Commun., 10, 5221.

Mathai,N. et al. (2019) Validation strategies for target prediction methods. Brief. Bioinform., bbz026, 112. 
Matter,H. (1997) Selecting optimally diverse compounds from structure databases: A validation study of two-dimensional and three-dimensional molecular descriptors. J. Med. Chem., 40, 1219-1229.

Nickel,J. et al. (2014) SuperPred: Update on drug classification and target prediction. Nucleic Acids Res., 42, 26-31.

Pribut,N. et al. (2019) Aryl substituted benzimidazolones as potent HIV-1 non-nucleoside reverse transcriptase inhibitors. ACS Med. Chem. Lett., 10, 196-202.

Qureshi,A. et al. (2018) HIV protI: an integrated web based platform for prediction and design of HIV proteins inhibitors. J. Cheminform., 10, 12.

Reker,D. et al. (2014) Identifying the macromolecular targets of de novo-designed chemical entities through self-organizing map consensus. Proc. Natl. Acad. Sci., 111, 4067-4072.

Rey,J. et al. (2019) PatchSearch: a web server for off-target protein identification. Nucleic Acids Res., 47, W365-W372.

Schenone,M. et al. (2017) Target identification and mechanism of action in chemical biology and drug discovery. Nat. Chem. Biol., 9, 232-240.

Stolbov,L. et al. (2019) AntiHIV-Pred: web-resource for in silico prediction of anti-HIV/AIDS activity. Bioinformatics.

Stone,M. (1976) Cross-validatory choice and assessment of statistical predictions. J. R. Stat. Soc. Ser. B, 38, 102-102.

Sydow,D. et al. (2019) Advances and challenges in computational target prediction. J. Chem. Inf. Model., 59, $1728-1742$.

Tai,H.K. et al. (2018) Chaos-embedded particle swarm optimization approach for protein-ligand docking and virtual screening. J. Cheminform., 10, 1-13. 
Vanhaelen,Q. et al. (2017) Design of efficient computational workflows for in silico drug repurposing. Drug Discov. Today, 22, 210-222.

Wainer,J. and Cawley,G. (2018) Nested cross-validation when selecting classifiers is overzealous for most practical applications. 1-9.

Wang,F. et al. (2019) ACID: a free tool for drug repurposing using consensus inverse docking strategy. $J$. Cheminform., 11, 73.

Wang,L. et al. (2013) TargetHunter: An in silico target identification tool for predicting therapeutic potential of small organic molecules based on chemogenomic database. AAPS J., 15, 395-406.

Wang,R. et al. (2004) The PDBbind database: collection of binding affinities for protein-ligand complexes with known three-dimensional structures. J. Med. Chem., 47, 2977-80.

Wang,X. et al. (2017) PharmMapper 2017 update: a web server for potential drug target identification with a comprehensive target pharmacophore database. Nucleic Acids Res., 45, W356-W360.

Wang,Z. et al. (2016) Improving chemical similarity ensemble approach in target prediction. $J$. Cheminform., 8, 1-10.

Wu,Z. et al. (2018) Network-based methods for prediction of drug-target interactions. Front. Pharmacol., 9, 1134.

Zhang,H. et al. (2019) Large-Scale Target Identification of Herbal Medicine Using a Reverse Docking Approach. ACS Omega, 4, 9710-9719. 